\title{
Thiazide Diuretic-Induced Change in Fasting Plasma Glucose: a Meta-analysis of Randomized Clinical Trials
}

\author{
Jill J. Hall, PharmD ${ }^{7} \mathbb{D}$, Dean T. Eurich, $P h D^{2}$, Danielle Nagy ${ }^{3}$, Lisa Tjosvold, MLIS , and \\ John-Michael Gamble, PhD 5
}

\begin{abstract}
'Faculty of Pharmacy and Pharmaceutical Sciences, 3-236 Edmonton Clinic Health Academy, University of Alberta, Edmonton, AB, Canada; ${ }^{2}$ School of Public Health, University of Alberta, Edmonton, Canada; ${ }^{3}$ Faculty of Pharmacy and Pharmaceutical Sciences, University of Alberta, Edmonton, Canada; ${ }^{4}$ John W. Scott Health Sciences Library, University of Alberta, Edmonton, Canada; ${ }^{5}$ School of Pharmacy, University of Waterloo, Kitchener, Canada.
\end{abstract}

\begin{abstract}
BACKGROUND: Prior meta-analyses measuring thiazideinduced glycemic change have demonstrated an increased risk of incident diabetes; however, this measure's definition has changed over time.
\end{abstract}

AIM: To determine the magnitude of change in fasting plasma glucose (FPG) for thiazide diuretics.

DATA SOURCES: A research librarian designed and conducted searches in Medline®, EMBASE, and EBM Reviews-Cochrane Central Register of Controlled Trials (inception through July 2018) and International Pharmaceutical Abstracts (inception to December 2014).

STUDY SELECTION: Randomized, controlled trials comparing a thiazide or thiazide-like diuretic to any comparator reporting FPG were identified. Trials enrolling $<50$ participants, those with a follow-up period of $<4$ weeks, and conference abstracts were excluded.

DATA EXTRACTION: Independent duplicate screening of citations and full-text articles, data extraction, and assessment of risk of bias was conducted.

DATA SYNTHESIS: Ninety-five studies were included ( $N=76,608$ participants), with thiazides compared with placebo, beta-blockers, calcium channel blockers, reninangiotensin-aldosterone-system inhibitors, potassiumsparing diuretic, and others alone or in combination. Thiazide diuretics marginally increased FPG (weighted mean difference $0.20 \mathrm{mmol} / \mathrm{L}(95 \%$ CI $0.15-0.25) ; I^{2}=84 \%$ ) ( $1 \mathrm{mmol} / \mathrm{L}=18 \mathrm{mg} / \mathrm{dL}$ ). Results did not change substantially when considering dose or duration, comparing thiazides with placebo or an active comparator, or using thiazides as monotherapy or combination therapy, even when combined with a potassium-correcting agent.

CONCLUSION: Thiazide diuretics have a small and clinically unimportant impact on FPG.

KEY WORDS: fasting plasma glucose; meta-analysis; randomized controlled trial; thiazide diuretic.

J Gen Intern Med 35(6):1849-60

DOI: $10.1007 / \mathrm{s} 11606-020-05731-3$

(c) Society of General Internal Medicine 2020

Electronic supplementary material The online version of this article (https://doi.org/10.1007/s11606-020-05731-3) contains supplementary material, which is available to authorized users.

Received October 14, 2019

Accepted February 10, 2020

Published online March 10, 2020

\section{INTRODUCTION}

Thiazide (e.g., hydrochlorothiazide) and thiazide-like (e.g., chlorthalidone, indapamide) diuretics (hereafter called "thiazide diuretics") are considered first-line therapy for the management of hypertension. ${ }^{1,2}$ However, the use of thiazide diuretics has been associated with concerns of adverse metabolic effects, including glycemic change and incident diabetes. $^{3,4}$

Thiazides are proposed to influence blood glucose by several mechanisms, including impaired insulin sensitivity, increased hepatic glucose production, and impaired peripheral uptake. ${ }^{5}$ Thiazides decrease serum potassium by $0.2-0.6 \mathrm{mEq} /$ $\mathrm{L}(1 \mathrm{mEq} / \mathrm{L}=1 \mathrm{mmol} / \mathrm{L})$ in a dose-dependent manner, ${ }^{3,} 6$ which has been shown retrospectively in aggregate to correlate with increased mean plasma glucose $;^{7}$ however, this has not been consistently demonstrated. A pre-specified analysis of the Pharmacogenomic Evaluation of Antihypertensive Responses (PEAR) study in 202 patients on hydrochlorothiazide $12.5-25 \mathrm{mg}$ for 9 weeks did not demonstrate a significant correlation between fasting plasma glucose (FPG) and potassium levels. ${ }^{8}$ Controversy also exists as to the potential impact of thiazide-induced glycemic change and incident diabetes on long-term adverse cardiovascular event rates and associated health care costs.

Previously published meta-analyses concerning thiazideinduced glycemic change have demonstrated an increased risk of incident diabetes ranging from an odds ratio of 1.16 to 1.30 as compared with placebo, calcium channel blockers (CCB), angiotensin-converting enzyme (ACE) inhibitors, and/or angiotensin receptor blockers (ARB). ${ }^{9-11}$ However, this measure's definition has changed over time; thus, we aimed to determine the magnitude of change in fasting plasma glucose (FPG).

\section{METHODS}

We conducted a systematic review with meta-analysis of the effects of thiazide and thiazide-like diuretics on changes in FPG according to PRISMA (Preferred Reporting Items for 
Systematic Reviews and Meta-Analyses) guidance on metaanalyses using a pre-specified study protocol. ${ }^{12}$

\section{Data Sources and Searches}

Randomized, controlled trials comparing a thiazide or thiazide-like diuretic with any comparator that did not fit this classification reporting any measure of blood glucose were identified. A research librarian designed and conducted searches in the following databases: Medline $\AA$, EMBASE, and EBM Reviews-Cochrane Central Register of Controlled Trials (between July 2012 and July 2018), as well as International Pharmaceutical Abstracts (up to December 2014 as the institutional subscription had been canceled). The detailed search strategy is described in eTable 1 . Searches were restricted using randomized controlled trial study design filters for each database, as well as to English language, humans, and adults. Reference lists of included studies were searched to identify any additional eligible trials and authors were contacted for additional information to enable study inclusion.

\section{Study Selection}

Randomized controlled trials comparing the effect of any thiazide diuretic at any dose as a first-line agent on fasting plasma glucose were included. We excluded trials enrolling less than 50 participants, those with a follow-up period of less than 4 weeks, and those reported only as conference abstracts; however, we did not restrict based on patient characteristics (age, sex, presence of hypertension, diabetes, or other metabolic disorders) or primary outcome measured. Studies evaluating children $(<18$ years) and those not in English were excluded. ${ }^{13,14}$ Authors of trials that mentioned a measure of glycemia but did not directly report data were contacted to acquire data where possible.

\section{Data Extraction and Quality Assessment}

Two reviewers screened the titles, abstracts, and subsequent full-text articles independently to identify relevant articles. Discrepancies were resolved by consensus or with consultation from a third reviewer if required. Data regarding patient population, intervention in the treatment and control group (drug, dose), study primary outcome, duration of follow-up, and baseline and endpoint measure of FPG were extracted and then independently verified, with discussion of any incongruity. FPG was extracted as mean and standard deviation at baseline and end of follow-up (to a maximum of 1 year [metabolic changes expected within 4 weeks]) where possible, and mean change and standard deviation of change within groups during the study period otherwise.

Two reviewers used the Cochrane tool to independently assess risk of bias within included trials. ${ }^{15}$ Selection bias (random sequence generation, allocation concealment), performance bias (blinding of participants and personnel), detection bias (blinding of outcome assessment), attrition bias (incomplete outcome data), and reporting bias (selective reporting) were rated as low, unclear, or high risk and used to provide an overall assessment of the trial's risk of bias.

\section{Data Synthesis and Analysis}

The primary outcome measure was the weighted mean difference (WMD) in FPG $(1 \mathrm{mmol} / \mathrm{L}=18 \mathrm{mg} / \mathrm{dL})$ comparing thiazide diuretic with any comparator, with a clinically important change defined as $0.4-0.5 \mathrm{mmol} / \mathrm{L}$, equating to a $0.3 \%$ change in A1c. ${ }^{16}$ In studies where more than one thiazide diuretic arm may have been evaluated (i.e., low versus high dose, or thiazide alone or in combination with other agents), data from the study arm with the highest dose utilized and thiazide use as monotherapy was preferred to maximize any potential signal related to glucose changes while also minimizing any confounding of the thiazide with other agents.

To summarize the effects of thiazide therapy on changes in FPG, we pooled estimates across studies using random effects models with inverse variance weighting (as recommended by Cochrane) as heterogeneity between the studies due to the difference in thiazide diuretic dose, comparator arms, and length of follow-up was expected. Heterogeneity was assessed using the $I^{2}$ statistic, with an $I^{2}$ statistic $>75 \%$ being considered as high heterogeneity. There was not an a priori degree of heterogeneity that precluded pooling. In studies that employed multiple comparison arms (e.g., a beta-blocker arm and a calcium channel blocker arm), a single combined comparator arm was created using the methods and formulas recommended by Cochrane for continuous data. ${ }^{15}$

In addition to the overall effect of thiazide diuretics, we a priori evaluated their effect in a number of subgroups based on comparator arm (placebo or any active comparator) as well as in specific active comparators (ACEi, ARB, or aliskiren; beta-blockers (BB); or CCB). An analysis of thiazides in combination with a potassium-correcting agent (potassium-sparing diuretic; ACEi, ARB, or aliskiren; or potassium supplement) was also conducted. Additional analyses according to thiazide dose (standard doses of thiazides defined as hydrochlorothiazide $\leq 25 \mathrm{mg}$, chlorthalidone $\leq 25 \mathrm{mg}$, indapamide $\leq 2.5 \mathrm{mg}$, trichlormethiazide $\leq 4 \mathrm{mg}$, altizide $\leq 15 \mathrm{mg}$, bendroflumethiazide $\leq 2.5 \mathrm{mg}$, hydroflumethiazide $\leq 25 \mathrm{mg}$ ) were also explored in subgroup and meta-regression approaches.

After initial pooling of study results, 3 additional sensitivity analyses were completed post hoc to explore the impact of specific studies on the estimates or to explore issues related to heterogeneity. Specifically, we evaluated the effect of thiazides overall in studies of a $<6$-month duration and in those with $\geq$ 6-month duration. Second, we removed several studies that appeared to be outliers based on the magnitude of effect observed (positive or negative). Specifically studies by Foder et al., Cicero et al., and Rubio-Guerra et al. were excluded in these analyses. ${ }^{17-19}$ Finally, we removed the 2 largest studies, Antihypertensive and Lipid-Lowering Treatment to Prevent 
Heart Attack Trial (ALLHAT) and Avoiding Cardiovascular Events through Combination Therapy in Patients Living with Systolic Hypertension (ACCOMPLISH), which had the most weight in the pooled results and repeated the analysis. ${ }^{20,21}$

Publication bias was assessed via visual inspection of a funnel plot and the use of an Egger test. All analyses were conducted using Stata MP Version 15 (College Station, TX 77845).

\section{RESULTS}

\section{Study Characteristics}

In total, 13,343 records were identified, with 7674 screened by title and abstract after removal of duplicates and 1692 full-text articles assessed for eligibility (Fig. 1). Of these, 1597 were excluded, the majority because they did not report fasting glucose data. Overall, 95 studies were included, all but 2 of which enrolled patients with hypertension, with a total of 76,608 participants $(33,357$ of whom were enrolled in ALLHAT). The mean age of participants was 58 years (range of the means $43-74$ years) and on average, 55\% of the participants were male. Participants were followed for a mean of 35 weeks (range of the means 4-260 weeks).

Thiazides were compared with the placebo ( $n=15$ comparisons $),{ }^{22-36} \mathrm{BB}(n=13),{ }^{37-49} \mathrm{CCB}(n=12),{ }^{17}, 20,25,39,42,48$, ${ }^{50-55}$ RAASi $(n=28),{ }^{20,} 23,39,42,48,50,56-74$ potassium-sparing diuretic $(n=4)^{75-78}$ or others (none, diet, alpha-blocker, loop diuretic, sodium glucose cotransporter-2 inhibitors) $(n=10)^{18}$, 79-87 alone or to any combination $(n=30)^{19}, 21,52,88-111$ (Table 1, eTable 2). Thiazide diuretics were commonly utilized as monotherapy $(n=59), 17,18,20,23,25-28,30-35,38-45,47-49,51$, $53-55,57,59,61,63-65,67,68,70,72,73,75-86,102$ dual therapy (with $\mathrm{BB}(n=4),{ }^{43,84,91,101} \mathrm{CCB}(n=1),{ }^{25} \mathrm{RAASi}(n=36),{ }^{19,21,23}$ $46,52,60,62,65,68,69,71,88,90,92-97,99,100,103,105-108,110,111$

potassium-sparing diuretics $(n=12), 22,24,28,29,58,66,74-77,81,87$ potassium supplements $(n=4)),{ }^{37,50,98,117}$ and other combi-

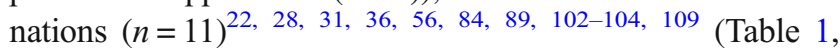
eTable 2).

Trials were generally judged to be of low to moderate risk of bias (Fig. 2, eTable 3). Few trials were at high risk of bias for blinding of participants, personnel, and/or assessors (14 to $29 \%$ ) or selective reporting (1\%); a large number had unclear risk of bias for random sequence generation $(66 \%)$ and/or allocation concealment (77\%).

\section{Effect of Thiazides on Fasting Plasma Glucose}

Overall, in pooled analyses, thiazide diuretics were found to marginally increase FPG (WMD $0.20 \mathrm{mmol} / \mathrm{L}$ (95\% CI 0.15 $0.25)$ ) (Fig. 3, eFigure 1). Heterogeneity was relatively high $\left(I^{2}=84 \%, p<0.001\right)$ and decreased with the removal of the 3 outlying studies (WMD $0.16 \mathrm{mmol} / \mathrm{L}$ (95\% CI $0.12-0.21$ ), $\left.I^{2}=61 \%\right){ }^{17-19}$ A sensitivity analysis removing ALLHAT ${ }^{20}$ and ACCOMPLISH, ${ }^{21}$ the 2 largest studies, did not impact the results (WMD $0.21 \mathrm{mmol} / \mathrm{L}(95 \%$ CI $0.15-0.26), I^{2}=83 \%$ ) (Fig. 3). Assessment of the funnel plots indicated asymmetrical suggesting the presence of publication bias (Eggers test $p<0.001$ ) (eFigure 2).

A subgroup analysis of thiazides utilized as monotherapy and as combination therapy produced similar results (WMD $0.17 \mathrm{mmol} / \mathrm{L}$ (95\% CI $0.10-0.24$ ), $I^{2}=83 \%$ (eFigure 3 ) and WMD $0.23 \mathrm{mmol} / \mathrm{L}\left(95 \%\right.$ CI 0.14-0.32), $I^{2}=80 \%$ (eFigure 4), respectively). Studies evaluating a duration of treatment $\geq$ 6 months (WMD $0.15 \mathrm{mmol} / \mathrm{L}$ (95\% CI $0.09-0.20), I^{2}=$ $68 \%$ ) (eFigure 5) and < 6 months (WMD $0.29 \mathrm{mmol} / \mathrm{L}(95 \%$ CI 0.19-0.40), $I^{2}=87 \%$ ) (eFigure 6) also demonstrated a similar impact on FPG.

In analyzing studies comparing thiazides with placebo versus an active comparator (of any type), again, a similar impact on FPG was seen (WMD $0.16 \mathrm{mmol} / \mathrm{L}$ (95\% CI 0.07-0.25), $I^{2}=72 \%$ (eFigure 7) and WMD $0.21 \mathrm{mmol} / \mathrm{L}$ (95\% CI 0.15 $0.28), I^{2}=84 \%$ (eFigure 8 ), respectively). Sensitivity analyses of thiazides versus various drug classes (ACEi, ARB, or aliskiren; beta-blockers; and calcium channel blockers) were similar (eFigures 9-11), as were those in populations with diabetes (WMD $0.24 \mathrm{mmol} / \mathrm{L}$ (95\% CI 0.07-0.41), $I^{2}=$ $75 \%$ ) (eFigure 12) and no history of diabetes or impaired FPG (WMD $0.25 \mathrm{mmol} / \mathrm{L}(95 \%$ CI $0.15-0.36), I^{2}=80 \%$ ) (eFigure 13).

An analysis of thiazide dose was also conducted, with standard and higher doses of thiazides demonstrating similar effects on FPG: WMD $0.21 \mathrm{mmol} / \mathrm{L}$ (95\% CI 0.14-0.28), $I^{2}=$ $80 \%$ (eFigure 14 ) and WMD $0.18 \mathrm{mmol} / \mathrm{L}$ (95\% CI 0.09 0.26 ), $I^{2}=80 \%$ (eFigure 15), respectively. Meta-regression utilizing the weighted average for FPG and the equivalent of maximum total daily dose hydrochlorothiazide also did not demonstrate a significant relationship $(p>0.05$, data not shown).

Finally, an analysis of thiazides in combination with a potassium-correcting agent (ACEi, ARB, or aliskiren, potassium-sparing diuretic, or potassium supplement) was conducted (WMD $0.23 \mathrm{mmol} / \mathrm{L}$ (95\% CI $0.13-0.33), I^{2}=$ $81 \%$ (eFigure 16)).

\section{DISCUSSION}

This meta-analysis (97 comparisons across 95 trials) demonstrated a statistically significant but clinically unimportant increase in FPG. Overall, heterogeneity in this main result was high and despite numerous subgroup and sensitivity analyses was not identifiable but was likely due to features of the study design (dose, duration of treatment, co-intervention) or population (diabetes/not, comorbidities), ${ }^{119}$ rather than true outliers given their removal did not alter the result.

The magnitude of the change in FPG with thiazide diuretics was similar across subgroups and among studies utilizing standard doses, some form of potassium correction (RAAS inhibitor, potassium-sparing diuretic, or potassium 

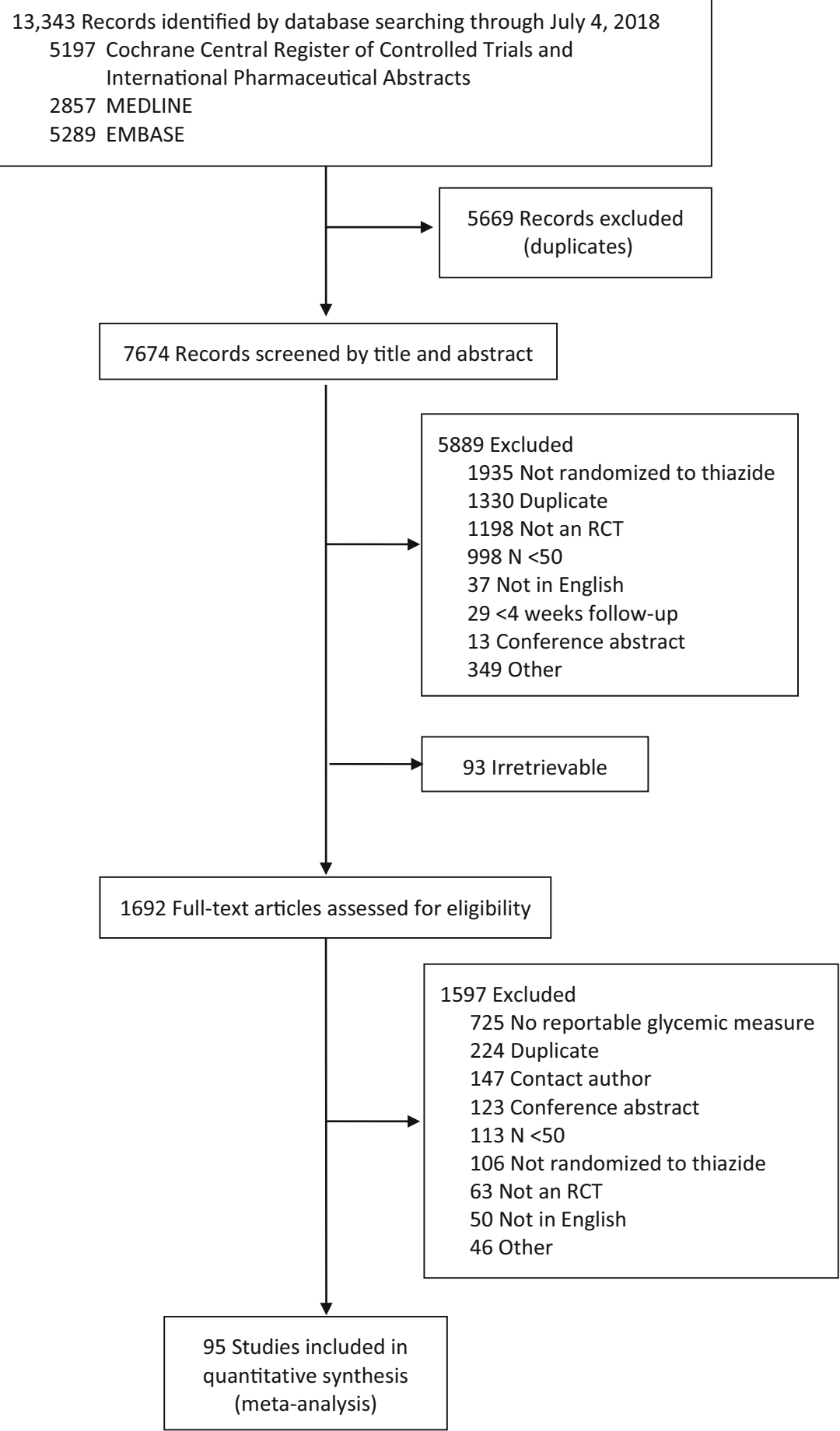

Figure 1 Summary of study retrieval and identification for meta-analysis.

supplement), and a thiazide in combination with any other drug. Although we had anticipated that the impact of standard doses of thiazide diuretics may be less than higher doses, given that they have been shown to impact potassium to a lesser degree, ${ }^{120}$ our data suggested otherwise with respect to changes in FPG. However, in our meta-analysis, there was no clinically important change in FPG when considering subgroups or other agents that may potentially change potassium levels. Given that we did not specifically evaluate change in
FPG in relation to potassium, the potential for a relationship to exist remains; our data, however, would suggest the clinical impact at the population level on FPG is negligible.

For this analysis, a follow-up was truncated at 1 year recognizing that most metabolic alterations occur during the first several weeks of therapy and most incident diabetes within the first year. ${ }^{8,}{ }^{121}$ While the magnitude of the effect for studies with a longer duration of treatment ( $>6$ months compared with those less than 6 months) appears to be less, this result is most likely 
Table 1 Selected Characteristics of Included Studies

\begin{tabular}{|c|c|c|c|c|}
\hline $\begin{array}{l}\text { First author (year of } \\
\text { publication) }\end{array}$ & $\begin{array}{l}\text { Intervention arm (maximum total } \\
\text { daily dose (mg)) }\end{array}$ & $\begin{array}{l}\text { Control arm (maximum } \\
\text { total daily dose }(\mathrm{mg}))\end{array}$ & $\begin{array}{l}\text { Nin } \\
\text { primary } \\
\text { analysis }\end{array}$ & $\begin{array}{l}\text { Total } \\
\text { duration of } \\
\text { follow-up } \\
\text { (weeks) }\end{array}$ \\
\hline $\begin{array}{l}\text { ALLHAT Officers and } \\
\text { Coordinators }(2002)^{20} \text { [ALLHAT] }\end{array}$ & Chlorthalidone 25 & $\begin{array}{l}\text { Amlodipine } 10 \text { or lisinopril } \\
40\end{array}$ & 12,819 & 104 \\
\hline Abe $(2009)^{71}$ & Hydrochlorothiazide $12.5+$ losartan 100 & Losartan 100 & 60 & 24 \\
\hline Amery (1986) [EWPHE] $]^{22}$ & $\begin{array}{l}\text { Hydrochlorothiazide } 50+\text { triamterene } 100 \pm \\
\text { methyldopa } 2000\end{array}$ & Placebo & 507 & 52 \\
\hline $\operatorname{Amin}(2015)^{79}$ & Hydrochlorothiazide 12.5 & Ertugliflozin 25 & 78 & 4 \\
\hline Bakris (2006) [STAR] $]^{105}$ & Hydrochlorothiazide $25+$ losartan 100 & $\begin{array}{l}\text { Verapamil SR } 240+ \\
\text { trandolapril } 4\end{array}$ & 184 & 52 \\
\hline Berglund (1981) 37 & Bendroflumethiazide $5+\mathrm{KCl} 15$ & Propranolol 320 & 106 & 52 \\
\hline Bichisao $(1984)^{38}$ & Chlorthalidone 25 & Metoprolol SR 200 & 738 & 4 \\
\hline Bosone $(2017)^{52}$ & Hydrochlorothiazide $25+$ ramipril 10 & $\begin{array}{l}\text { Amlodipine } 10 \text { or ramipril } \\
10+\text { canrenone } 100\end{array}$ & 289 & 52 \\
\hline Brandao $(2010)^{72}$ & Hydrochlorothiazide 25 or indapamide 1.5 & Perindopril 4 & 48 & 12 \\
\hline Brown (2016) ${ }^{75}$ [PATHWAY-3] & $\begin{array}{l}\text { hydrochlorothiazide } 50 \text { or hydrochlorothiazide } \\
25+\text { amiloride } 10\end{array}$ & Amiloride 20 & 166 & 24 \\
\hline Byington $(1998)^{53}$ [MIDAS] & Hydrochlorothiazide 50 & Isradipine 10 & 881 & 52 \\
\hline Calvo $(2000)^{54}$ & Hydrochlorothiazide 100 & Amlodipine 10 & 197 & 8 \\
\hline Campo $(2003)^{80}$ & Hydrochlorothiazide 25 & Doxazosin 8 & 98 & 16 \\
\hline Charansonney $(1997)^{81}$ [Eurevie] & $\begin{array}{l}\text { Hydrochlorothiazide } 25 \text { or altizide } 15+ \\
\text { spironolactone } 25\end{array}$ & Piretanide SR 6 & 599 & 24 \\
\hline Christogiannis $(2013)^{106}$ & Hydrochlorothiazide 12.5 + valsartan 160 & $\begin{array}{l}\text { Valsartan } 160+\text { amlodipine } \\
5\end{array}$ & 60 & 16 \\
\hline $\begin{array}{l}\text { Chrysant }(1994)^{23} \text { [The Lisinopril } \\
\text { Hydrochlorothiazide Group] }\end{array}$ & $\begin{array}{l}\text { Hydrochlorothiazide } 12.5 \text { or } \\
\text { hydrochlorothiazide } 25 \text { or hydrochlorothiazide } \\
12.5+\text { lisinopril } 10 \text { or hydrochlorothiazide } 25+ \\
\text { lisinopril } 10\end{array}$ & Placebo or lisinopril 10 & 165 & 8 \\
\hline Cicero $(2012)^{18}$ & Hydrochlorothiazide 12.5 & $\begin{array}{l}\text { Orthosiphon stamineus } \\
100\end{array}$ & 80 & 8 \\
\hline $\begin{array}{l}\text { Damian }(2016)^{29} ; \text { MRC Working } \\
\text { Party }(1992)^{112}[\text { MRC elderly } \\
\text { trial] }\end{array}$ & Hydrochlorothiazide $50+$ amiloride 5 & Placebo & 3294 & 52 \\
\hline Deedwania $(2013)^{107}$ & $\begin{array}{l}\text { Hydrochlorothiazide } 25+\text { (lisinopril } 10 \text { or } \\
\text { losartan 50) }\end{array}$ & $\begin{array}{l}\text { Placebo + (lisinopril } 10 \text { or } \\
\text { losartan } 50 \text { ) }\end{array}$ & 309 & 12 \\
\hline Derosa $(2015)^{108}$ & Hydrochlorothiazide $12.5+$ telmisartan 80 & $\begin{array}{l}\text { Losartan } 100+\text { barnidipine } \\
20\end{array}$ & 141 & 24 \\
\hline Ferdinand $(2011)^{109}$ [ASCENT] & $\begin{array}{l}\text { Hydrochlorothiazide } 25+\text { aliskiren } 300+ \\
\text { amlodipine } 10\end{array}$ & $\begin{array}{l}\text { Amlodipine } 10+\text { aliskiren } \\
300\end{array}$ & 411 & 8 \\
\hline Fiddes $(1997)^{30}$ & Indapamide 1.25 & Placebo & 188 & 8 \\
\hline Fodor $(1997)^{17}$ & Hydrochlorothiazide 50 & Nisoldipine 40 & 252 & 12 \\
\hline Fogari $(1995)^{42}$ & Hydrochlorothiazide 25 & $\begin{array}{l}\text { Amlodipine } 10 \text { or atenolol } \\
100 \text { or lisinopril } 20\end{array}$ & 118 & 8 \\
\hline Fogari $(2007)^{110}$ & Hydrochlorothiazide $25+$ candesartan 16 & $\begin{array}{l}\text { Manidipine } 20+ \\
\text { candesartan } 16\end{array}$ & 174 & 24 \\
\hline Fogari $(2008)^{111}$ & Hydrochlorothiazide $25+$ olmesartan 20 & $\begin{array}{l}\text { Manidipine } 20+\text { delapril } \\
30\end{array}$ & 150 & 48 \\
\hline Fogari $(2008)^{88}$ & Hydrochlorothiazide $12.5+$ olmesartan 20 & $\begin{array}{l}\text { Manidipine } 10+\text { delapril } \\
30\end{array}$ & 88 & 24 \\
\hline Fogari $(2014)^{89}$ & $\begin{array}{l}\text { Hydrochlorothiazide } 25+\text { valsartan } 160+ \\
\text { amlodipine } 5\end{array}$ & $\begin{array}{l}\text { Valsartan } 160+\text { amlodipine } \\
5+\text { canrenone } 50\end{array}$ & 109 & 24 \\
\hline Fonseca $(2015)^{73}$ & Hydrochlorothiazide 25 or indapamide 1.5 & Perindopril 4 & 44 & 12 \\
\hline Fuchs $(2015)^{74}$ [PREVER] & Chlorthalidone $25+$ amiloride 5 & Losartan 100 & 607 & 78 \\
\hline $\operatorname{Garg}(2015)^{56}$ & $\begin{array}{l}\text { Hydrochlorothiazide } 12.5+\mathrm{KCl} 10+\text { enalapril } \\
20\end{array}$ & Enalapril 20 & 41 & 24 \\
\hline Ghiadoni $(2017)^{90}$ & Hydrochlorothiazide $25+$ enalapril 20 & $\begin{array}{l}\text { Enalapril } 20+ \\
\text { lercanidipine } 20\end{array}$ & 76 & 24 \\
\hline $\begin{array}{l}\text { Goldman }(1980)^{31}[\text { Mild } \\
\text { Hypertension Feasibility Trial }]\end{array}$ & Chlorthalidone $100 \pm$ reserpine 0.25 & Placebo & 611 & 52 \\
\hline Grimm $(1981)^{32}$ & Hydrochlorothiazide 100 or chlorthalidone 100 & Placebo & 117 & 24 \\
\hline Haenni $(1994)^{57}$ & Bendrofluazide 5 & Lisinopril 20 & 61 & 24 \\
\hline Hegbrant (1989) 83 & Bendroflumethiazide 2.5 & $\begin{array}{l}\text { Piretanide SR } 6 \text { or } \\
\text { piretanide SR } 12\end{array}$ & 76 & 12 \\
\hline $\begin{array}{l}\text { Helgeland }(1984)^{84}(1980)^{113} \\
\text { [OSLO] }\end{array}$ & $\begin{array}{l}\text { Hydrochlorothiazide } 50 \pm \text { (propranolol } 320 \text { or } \\
\text { methyldopa 1500) }\end{array}$ & Untreated & 402 & 260 \\
\hline Holzgreve $(2003)^{91}$ & Chlorthalidone $25+$ atenolol 100 & $\begin{array}{l}\text { Verapamil SR } 180+ \\
\text { trandolapril } 2\end{array}$ & 450 & 20 \\
\hline Jounela $(1994)^{33}$ & Hydrochlorothiazide 3 or 6 or 12.5 or 25 & Placebo & 45 & 6 \\
\hline Karashima $(2016)^{92}$ & Hydrochlorothiazide $6.25+$ candesartan 8 & $\begin{array}{l}\text { Eplerenone } 50+ \\
\text { candesartan } 8\end{array}$ & 50 & 52 \\
\hline Kato $(2011)^{93}$ & $\begin{array}{l}\text { Olmesartan } 21.2^{*}+\left(\text { indapamide } 0.96^{*} \text { or }\right. \\
\text { trichlormethiazide } 1.47^{*} \text { ) }\end{array}$ & $\begin{array}{l}\text { Olmesartan } 22.1 *+ \\
\text { azelnidipine } 13.3 *+\end{array}$ & 58 & 24 \\
\hline
\end{tabular}


Table 1. (continued)

\begin{tabular}{lll}
\hline \hline $\begin{array}{l}\text { First author (year of } \\
\text { publication) }\end{array}$ & $\begin{array}{l}\text { Intervention arm (maximum total } \\
\text { daily dose }(\mathrm{mg}))\end{array}$ & $\begin{array}{l}\text { Control arm (maximum } \\
\text { total daily dose (mg)) }\end{array}$ \\
& & $\begin{array}{l}\text { Total in } \\
\text { primary } \\
\text { analysis } \\
\text { duration of } \\
\text { follow-up } \\
(w e e k s)\end{array}$ \\
\hline
\end{tabular}

Koh $(2010)^{34}$

Lambers Heerspink $(2013)^{35}$

Lee $(2012)^{94}$

Leehey $(1988)^{55}$

Lin $(1995)^{58}$

Lindholm $(2003)^{59}$ [ALPINE]

Makita $(2009)^{60}$

Malekzadeh $(2010)^{36}$

Maroko (1989) ${ }^{24}$ [PHICOG]

Marre (2004) ${ }^{61}$ [NESTOR]

Martinez-Martin (2011) ${ }^{95}$ [OLAS]

Matsui (2011) ${ }^{96}$ [J-CORE]

Milon (1984) ${ }^{44}$

Momeni (2015) ${ }^{77}$

Monmany (1990) ${ }^{45}$

Mugellini (2004) ${ }^{97}$

Multicenter Diuretic Cooperative Study Group (Douglas) $(1981)^{76}$

Nielsen $(1994)^{98}$

Nishimura $(2013)^{62}$

Nishiwaki $(2013)^{99}$

Obel $(1984)^{85}$

Os $(1997)^{4}$

Oshikawa (2014) ${ }^{100}$

Pareek (2008) $)^{101}$

Pollare $(1989)^{63}$

Pollavini $(1984)^{47}$

Pool $(1993)^{25}$

Posadzy-Malaczynska $(2015)^{102}$

Rajzer (2017) ${ }^{48}$

Rasmussen $(2006)^{50}$

Reid $(2000)^{26}$

Roman $(1998)^{64}$

Rosenthal $(1990)^{65}$

Rubio-Guerra (2017) ${ }^{19}$, Duran-

Salgado (2015) ${ }^{114}$

Saruta $(2015)^{103}$, Ogihara

$(2014)^{115}$ [COLM]

Savage (1998) ${ }^{27}$, SHEP

Cooperative Research Group

$(1991)^{116}$ [SHEP]

Scali $(1992)^{66}$

Siegel $(1992)^{117},(1994)^{28}$

Skoczylas $(2016)^{39}$
Hydrochlorothiazide 50

Hydrochlorothiazide 25

Hydrochlorothiazide 25 + valsartan 160

Hydrochlorothiazide 75

Hydrochlorothiazide $50+$ amiloride 5

Hydrochlorothiazide 25

Hydrochlorothiazide 12.5 + telmisartan 40

Hydrochlorothiazide 12.5 + ASA 81 + enalapril

$2.5+$ atorvastatin 20

Hydrochlorothiazide $25+$ triamterene 50

Indapamide SR 1.5

Hydrochlorothiazide $25+$ olmesartan 40

Hydrochlorothiazide 12.5 + olmesartan 20

Chlorthalidone 100

Hydrochlorothiazide 25 or hydrochlorothiazide

$25+$ spironolactone 50

Hydrochlorothiazide 100

Hydrochlorothiazide 12.5 + irbesartan 150

Hydrochlorothiazide 100 or hydrochlorothiazide $100+$ amiloride 10

Bendroflumethiazide $5+\mathrm{KCl} 15$

Hydrochlorothiazide $12.5+$ losartan 50

Hydrochlorothiazide 12.5 + losartan 100

Bendroflumethiazide 10

Hydrochlorothiazide $6+$ enalapril 20

Hydrochlorothiazide $12.5+$ losartan 100

Chlorthalidone $6.25+$ atenolol 25

Hydrochlorothiazide 50

Chlorthalidone 25

Hydrochlorothiazide 12.5 + diltiazem SR 120 or hydrochlorothiazide 12.5

Hydrochlorothiazide $25 \pm$ estrogen progesterone therapy

Hydrochlorothiazide 50

Bendroflumethiazide $1.25+\mathrm{KCl} 7.6$ or

bendroflumethiazide $2.5+\mathrm{KCl} 7.6$

Hydrochlorothiazide 50

Hydrochlorothiazide 50

Hydrochlorothiazide 25 or hydrochlorothiazide

$25+$ enalapril 40

Hydrochlorothiazide $12.5+$ losartan 100

Olmesartan $40+$ (indapamide 1 or

trichlormethiazide 1 or hydrochlorothiazide 12 or benzylhydrochlorothiazide 2 or meticrane 75 or tripamide 7.5 or chlorthalidone 25 or

mefruside 12.5)

Chlorthalidone 25

Hydrochlorothiazide $100+$ amiloride 10

Hydrochlorothiazide 50 or hydrochlorothiazide

$50+\mathrm{KCl} 40$ or hydrochlorothiazide $50+\mathrm{KCl}$

$40+$ magnesium oxide or hydrochlorothiazide

$50+$ triamterene 100 or chlorthalidone 50

Indapamide 3 (amlodipine $3.5^{*}$ or

benidipine $4 *$ )

Placebo

Placebo

Amlodipine $10+$

benazepril 20

Diltiazem SR 360

51

Captopril 150 or enalapril 40

Candesartan 16

Candesartan 8 or valsartan 80

Placebo 474

$62-8$

61

52

$392 \quad 52$

$64 \quad 12$

$474 \quad 52$

Placebo

Enalapril 10

Olmesartan $40+$

amlodipine 10

Olmesartan $20+$

azelnidipine 16

Atenolol 100

Spironolactone 50

352

510

120

207

67

40

Metoprolol 300

Delapril $30+$ manidipine 10

Amiloride 10

37

80

90

Enalapril $20+\mathrm{KCl} 15$

114

Losartan 100

106

Losartan $100+$ amlodipine

5

Furosemide SR 60

Atenolol 50

Amlodipine $5+$ losartan 100

Amlodipine $2.5+$ (atenolol

25 or atenolol 50)

Captopril 100

Oxprenolol SR 160

Placebo or diltiazem SR

120

Perindopril $4 \pm$ estrogen

progesterone therapy

Quinapril 40 or amlodipine

10 or losartan 100 or

bisoprolol 10

Amlodipine 5 or enalapril

10

Placebo

Ramipril 20

Enalapril 40

Amlodipine $5+$ losartan

100

Olmesartan $40+$

(azelnidipine 16 or

amlodipine 5)

Placebo

2807

Lisinopril 20

62

Placebo

55

Bisoprolol 10 or amlodipine 10 or candesartan 32
24

0

52

2

8

78

24

24

12

52

12

20

48

36

12 
Table 1. (continued)

\begin{tabular}{|c|c|c|c|c|}
\hline $\begin{array}{l}\text { First author (year of } \\
\text { publication) }\end{array}$ & $\begin{array}{l}\text { Intervention arm (maximum total } \\
\text { daily dose }(\mathrm{mg}) \text { ) }\end{array}$ & $\begin{array}{l}\text { Control arm (maximum } \\
\text { total daily dose (mg)) }\end{array}$ & $\begin{array}{l}N \text { in } \\
\text { primary } \\
\text { analysis }\end{array}$ & $\begin{array}{l}\text { Total } \\
\text { duration of } \\
\text { follow-up } \\
\text { (weeks) }\end{array}$ \\
\hline Smith $(2012)^{40}[$ PEAR $]$ & Hydrochlorothiazide 25 & Atenolol 100 & 569 & 9 \\
\hline Stimpel $(1998)^{67}$ & Hydrochlorothiazide 25 & Moexipril 15 & 97 & 12 \\
\hline Takihata $(2015)^{51}[\mathrm{COAT}]$ & Trichlormethiazide 1 & Azelnidipine 16 & 209 & 48 \\
\hline Tani $(2017)^{104}$ & Indapamide $1+$ amlodipine $5+$ irbesartan 100 & $\begin{array}{l}\text { Amlodipine } 10+\text { irbesartan } \\
100\end{array}$ & 115 & 12 \\
\hline Ueda $(2014)^{86}[\mathrm{DIME}]$ & $\begin{array}{l}\text { Hydrochlorothiazide } 12.5 \text { or indapamide } 1 \text { or } \\
\text { trichlormethiazide } 1\end{array}$ & $\begin{array}{l}\text { Any antihypertensive other } \\
\text { than thiazides }\end{array}$ & 1130 & 229 \\
\hline $\begin{array}{l}\text { Veterans Cooperative Study } \\
\text { Group on Antihypertensive } \\
\text { Agents (Freis } 1979)^{82}\end{array}$ & Hydrochlorothiazide 200 & Ticrynafen 1000 & 155 & 30 \\
\hline $\begin{array}{l}\text { Veterans Cooperative Study } \\
\text { Group on Antihypertensive }\end{array}$ & $\begin{array}{l}\text { Bendroflumethiazide } 10 \text { or bendroflumethiazide } \\
10+\text { nadolol } 240\end{array}$ & Nadolol 240 & 164 & 12 \\
\hline $\begin{array}{l}\text { Agents (Freeis } 1983)^{43} \\
\text { Veterans Cooperative Study } \\
\text { Group on Antihypertensive } \\
\text { Agents (Ramirez 1985) } \\
1982)^{118} \text { (Freis }\end{array}$ & Hydrochlorothiazide 200 & Propranolol 640 & 294 & 58 \\
\hline Weber $(2010)^{21}$ [ACCOMPLISH] & Hydrochlorothiazide $25+$ benazepril 40 & $\begin{array}{l}\text { Benazepril } 40+ \\
\text { amlodipine } 10\end{array}$ & 6946 & 130 \\
\hline Wicker $(1986)^{87}$ & Hydrochlorothiazide $50+$ amiloride 5 & Muzolimine 20 & 47 & 20 \\
\hline Yonga $(1993)^{41}$ & Hydroflumethiazide 50 & Propranolol 160 & 53 & 12 \\
\hline Yutaka $(2009)^{78}$ & Trichlormethiazide $0.857 *$ & Spironolactone $10.7 *$ & 64 & 24 \\
\hline Zappe $(2008)^{68}$ [MADE-ITT] & $\begin{array}{l}\text { Hydrochlorothiazide } 25 \text { or hydrochlorothiazide } \\
25+\text { valsartan } 320\end{array}$ & Valsartan 320 & 363 & 16 \\
\hline Zhang $(2010)^{69}$ & Indapamide $2.5+$ fosinopril 20 & Fosinopril 20 & 89 & $52-64$ \\
\hline Zhang $(2017)^{70}$ & Hydrochlorothiazide 25 & Telmisartan 40 & 1023 & 8 \\
\hline
\end{tabular}

Note. $\mathrm{KCl}$, potassium chloride ( $\mathrm{mEq}$ ); SR, sustained or slow release formulation; +, "and"; \pm , "and/or" as additional or step up therapy after initial randomization

* Mean dose per day

due to chance given the overlapping confidence intervals and differences in study design and population, as noted above.

Baseline blood glucose has been associated with changes in blood glucose or incident diabetes with thiazide therapy. A PEAR study analysis (hydrochlorothiazide $25 \mathrm{mg}$ versus atenolol $100 \mathrm{mg}$ ) demonstrated that baseline blood glucose was the greatest predictor of change in blood glucose or incident impaired fasting glucose, ${ }^{122}$ similar to that reported in the PIUMA hypertension registry, where baseline impaired FPG, higher 24$\mathrm{h}$ ambulatory blood pressure, and (low-dose) thiazide diuretic use predicted incident diabetes. ${ }^{123}$ Moreover, in an analysis of hydrochlorothiazide impact on FPG in patients with type 2 diabetes, Lin et al. also observed an increased in FPG (standardized mean difference (SMD) 0.27 (95\% CI 0.11-0.43)). ${ }^{124}$ These data are aligned with our results; however, there was no appreciable difference in the magnitude of change in patients with and without pre-existing diabetes or impaired FPG. Recognizing that this change may have different meaning to patients in either group, notably no change in FPG reached the $0.5 \mathrm{mmol} / \mathrm{L}$ change threshold for clinical importance. ${ }^{16}$

In comparison with a recently published meta-analysis also investigating the association of thiazide diuretics with

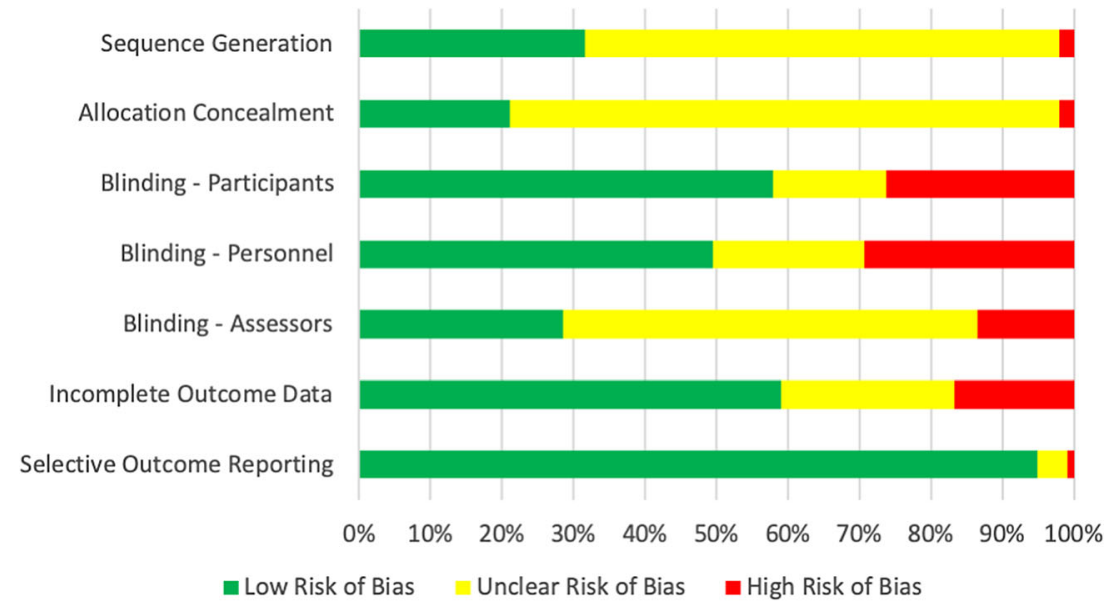

Figure 2 Summary of risk of bias for trials included in meta-analysis. 


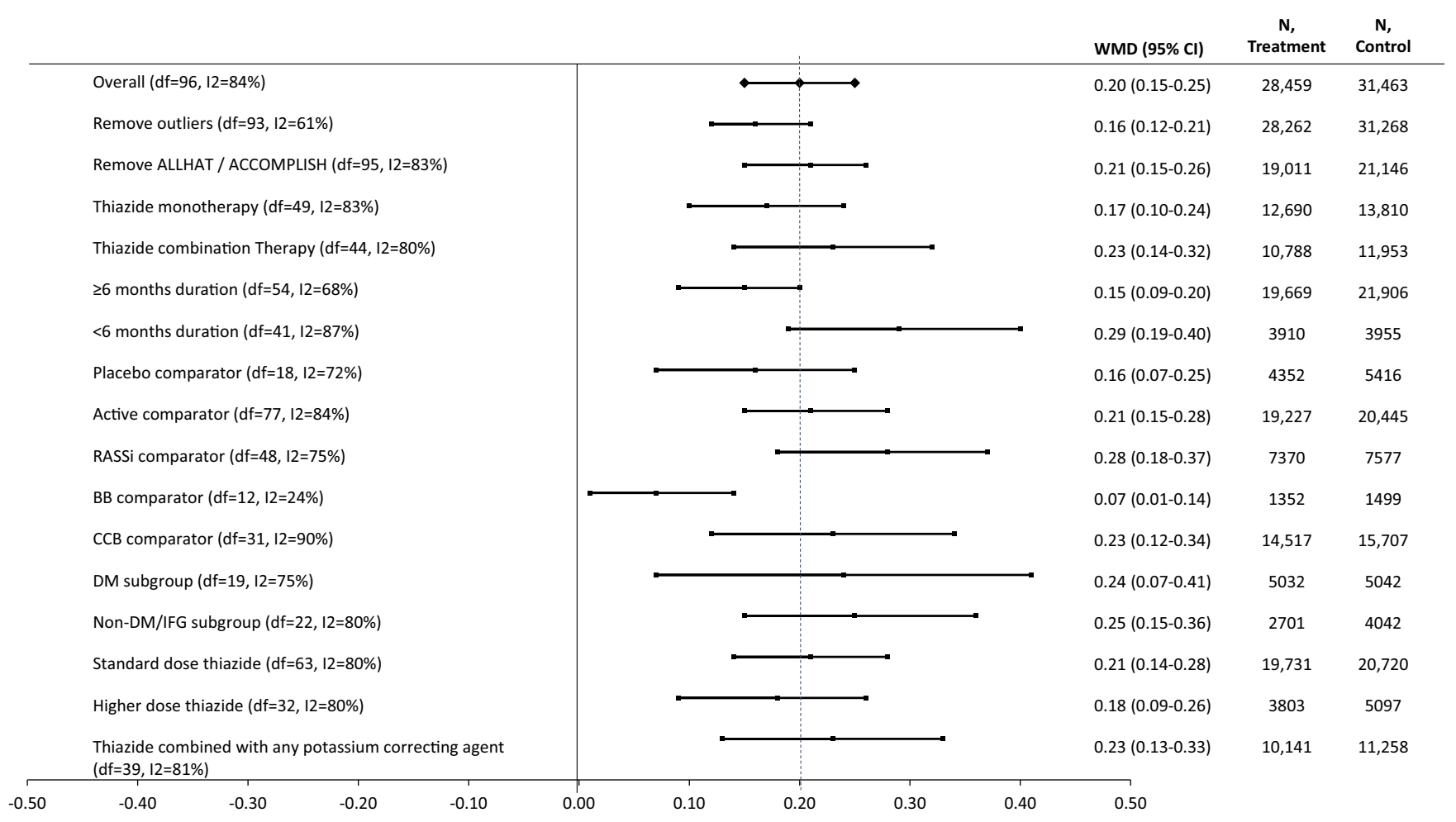

Figure 3 Summary of forest plot demonstrating the weighted mean difference in fasting blood glucose $(1 \mathrm{mmol} / \mathrm{L}=18 \mathrm{mg} / \mathrm{dL}) \mathrm{in}$ patients treated with thiazide diuretics overall and in sensitivity and subgroup analyses.

glycemic changes in randomized controlled clinical trials, our study includes a larger number of trials and participants. ${ }^{125}$ Zhang et al. concluded that thiazide-type diuretics produce a statistically significant increase in FPG (mean difference (MD) $0.27 \mathrm{mmol} / \mathrm{L}$ (95\% CI 0.15-0.39)), which was mitigated in the lower dose analysis (MD $0.15 \mathrm{mmol} / \mathrm{L}$ (95\% CI 0.03-0.27)) and with time (studies $<6$ months MD $0.5 \mathrm{mmol} / \mathrm{L}$, compared with studies $>6$ months $\mathrm{MD}-0.01 \mathrm{mmol} / \mathrm{L}$ ). Although all 26 included trials were captured in our search, only 15 were included in our analysis primarily for follow-up and sample size reasons. Despite this, the larger number of participants in our analysis and the broader breadth of patients included allowed for a more comprehensive assessment of thiazide use on FPG, particularly among important subgroups of patients not previously assessed in the Zhang et al. paper.

There are several further strengths of our study. We were more inclusive of trial populations as compared with other systematic reviews, and with the analyses including data from 95 trials and 76,608 participants, this is the largest meta-analysis addressing this question. Concerns over the use of a weighted mean difference may exist; however, an analysis of SMD was completed, which allows for the expression of the effect size of the thiazide diuretics in each study relative to its variability despite the inconsistency in study populations and treatment strategies - results were unchanged (SMD $0.17 \mathrm{mmol} / \mathrm{L}$ (95\% CI 0.12-0.21)). Utilizing FPG rather than new-onset diabetes as an outcome is also a strength, in that the latter is plagued by differing definitions over time and is limited by its low incidence and the short followup in individual trials. The use of FPG also enables the inclusion of results for a larger proportion of the study population and is relatively quickly responsive to any influencers; however, it was rarely the study primary outcome, and a one-time unconfirmed measurement may not be accurate and/or reflective of average blood glucose levels. Last, imbalance with respect to studies with large weighted mean differences was observed, which may be due to reporting bias, poor methodological quality, true heterogeneity, or chance. ${ }^{126}$ As a result, our results may overestimate the effect of thiazides of FPG.

Our study is not without limitations. Given the timespan over which included studies were conducted, it was not reasonable to analyze patient-level data nor were we successful in obtaining study data (except for 1 article $^{73}$ ) where papers indicated a measure of glucose without necessary details (authors were either not contactable or no longer had data of interest). The design of included studies also did not enable an analysis of confounding by co-intervention, outside of those which were assigned at randomization. Finally, we excluded non-English papers, deemed reasonable given reports that excluding non-English studies does not influence results of meta-analyses, ${ }^{13}, 14$ and results reported only as conference proceedings, and could not access the IPA database after 2014, which may have further limited our dataset.

\section{CONCLUSION}

Thiazide diuretics have a small and clinically unimportant impact on FPG. 
Acknowledgments: We would like to acknowledge the following contributors, without whom this work would not have been possible: Katelyn Crick and Amanda Lau, students in the School of Public Health, and Peter Yang, Daniel Zhou, Danielle Portnoy, Kevin Jacobson, and Salwa Said, students in the Faculty of Pharmacy and Pharmaceutical Sciences.

Corresponding Author: Jill J. Hall, PharmD; Faculty of Pharmacy and Pharmaceutical Sciences, 3-236 Edmonton Clinic Health Academy University of Alberta, Edmonton, AB, Canada (e-mail:jhall@ualberta.ca).

\section{Compliance with Ethical Standards:}

Conflict of Interest: No author has any financial arrangements or potential conflicts of interest to disclose with regard to products in this manuscript.

\section{REFERENCES}

1. Nerenberg KA, Zarnke KB, Leung AA, et al. Hypertension Canada's 2018 Guidelines for Diagnosis, Risk Assessment, Prevention, and Treatment of Hypertension in Adults and Children. Can $J$ Cardiol. 2018;34(5):506-525. doi:https://doi.org/10.1016/j.cjca.2018.02.022

2. James PA, Oparil S, Carter BL, et al. Evidence-Based Guideline for the Management of High Blood Pressure in Adults. JAMA. 2014;311(5):507520. doi:https://doi.org/10.1001/jama.2013.284427

3. Carter BL, Einhorn PT, Brands M, et al. Thiazide-induced dysglycemia: Call for research from a working group from the national heart, lung, and blood institute. Hypertension. 2008;52(1):30-36. doi:https:// doi.org/10.1161/HYPERTENSIONAHA. 108.114389

4. Elliott WJ. Effects of potassium-sparing versus thiazide diuretics on glucose tolerance: New data on an old topic. Hypertension. 2012;59(5):911-912. doi:https://doi.org/10.1161/HYPERTENSIONAHA.112.192542

5. Eriksson JW, Jansson PA, Carlberg B, et al. Hydrochlorothiazide, but not candesartan, aggravates insulin resistance and causes visceral and hepatic fat accumulation: The Mechanisms for the Diabetes Preventing Effect of Candesartan (MEDICA) study. Hypertension. 2008;52(6):1030 1037. doi:https://doi.org/10.1161/HYPERTENSIONAHA. 108.119404

6. Duarte JD, Cooper-Dehoff RM. Mechanisms for blood pressure lowering and metabolic effects of thiazide and thiazide-like diuretics. Expert Rev Cardiovasc Ther. 2010;8(6):793-802.

7. Zillich AJ, Garg J, Basu S, Bakris GL, Carter BL. Thiazide diuretics, potassium, and the development of diabetes: A quantitative review. Hypertension. 2006;48(2):219-224. doi:https://doi.org/10.1161/01. HYP.0000231552.10054.aa

8. Smith SM, Anderson SD, Wen S, et al. Lack of correlation between thiazide-induced hyperglycemia and hypokalemia: subgroup analysis of results from the pharmacogenomic evaluation of antihypertensive responses (PEAR) study. Pharmacotherapy. 2009;29(10):1157-1165. doi:https://doi.org/10.1592/phco.29.10.1157

9. Kuti EL, Baker WL, White CM. The development of new-onset type 2 diabetes associated with choosing a calcium channel blocker compared to a diuretic or beta-blocker. Curr Med Res Opin. 2007;23(6):12391244. doi:https://doi.org/10.1185/030079907X188044

10. Mason JM, Dickinson HO, Nicolson DJ, Campbell F, Ford GA, Williams B. The diabetogenic potential of thiazide-type diuretic and beta-blocker combinations in patients with hypertension. $J$ Hypertens 2005;23(10): 1777-1781.

11. Elliott WJ, Meyer PM. Incident diabetes in clinical trials of antihypertensive drugs: a network meta-analysis. Lancet. 2007;369(9557):201207. doi:https://doi.org/10.1016/S0140-6736(07)60108-1

12. Liberati A, Altman DG, Tetzlaff J, Mulrow C, Gøtzsche PC, John PA The PRISMA statement for reporting systematic reviews and metaanalyses of studies that evaluate healthcare interventions: explanation and elaboration. BMJ Online 2009;339:b2700. doi:https://doi.org/10. 1136/bmj.b2700

13. Morrison A, Polisena J, Husereau D, et al. The effect of Englishlanguage restriction on systematic review-based meta-analyses: A systematic review of empirical studies. Int $J$ Technol Assess Health Care.
$2012 ; 28(2): 138-144$. doi:https://doi.org/10.1017/ S0266462312000086

14. Moher D, Pham B, Klassen TP, et al. What contributions do languages other than English make on the results of meta-analyses? J Clin Epidemiol. 2000;53(9):964-972. doi:https://doi.org/10.1016/S08954356(00)00188-8

15. Higgins J, Green S, eds. Cochrane Handbook for Systematic Reviews of Interventions Version 5.1.1 [Updated March 2011]. The Cochrane Collaboration, 2011. www.handbook.cochrane.org.

16. Maruthur NM, Tseng E, Hutfless S, et al. Diabetes medications as monotherapy or metformin-based combination therapy for type 2 diabetes: A systematic review and meta-analysis. Ann Intern Med. 2016;164(11):740-751. doi:https://doi.org/10.7326/M15-2650

17. Fodor J. Comparative efficacy and tolerability of nisoldipine coat core and hydrochlorothiazide in mild-to-moderate hypertension. Int $J$ Clin Pract. 1997;51(5):271-275.

18. Cicero AFG, De Sando V, Izzo R, Vasta A, Trimarco A, Borghi C. Effect of a combined nutraceutical containing Orthosiphon stamineus effect on blood pressure and metabolic syndrome components in hypertensive dyslipidaemic patients: A randomized clinical trial. Complement Ther Clin Pract. 2012;18:190-194. doi:https://doi.org/10.1016/j.ctcp.2012. 02.002

19. Rubio-Guerra AF, Garro-Almendaro AK, Elizalde-Barrera CI, SuarezCuenca JA, Duran-Salgado MB. Effect of losartan combined with amlodipine or with a thiazide on uric acid levels in hypertensive patients. Ther Adv Cardiovasc Dis. 2017;11(2):57-62. doi:https://doi. org/10.1177/1753944716678538

20. "The ALLHAT Officers and Coordinators for the ALLHAT Collaborative Research Group." Major Outcomes in High-Risk Hypertensive Patients Randomized to Angiotensin-Converting Enzyme Inhibitor or Calcium Channel Blocker vs Diuretic. JAMA. 2002;288(23):2981-2196. http:// jama.jamanetwork.com/.

21. Weber MA, Bakris GL, Jamerson $\mathbf{K}$, et al. Cardiovascular Events During Differing Hypertension Therapies in Patients With Diabetes. $J$ Am Coll Cardiol. 2010;56(1):77-85. doi:https://doi.org/10.1016/j.jacc. 2010.02.046

22. Amery A, Birkenhäger W, Brixko $\mathbf{P}$, et al. Glucose intolerance during diuretic therapy in elderly hypertensive patients. A second report from the European Working Party on high blood pressure in the elderly (EWPHE). Postgrad Med J. 1986;62:919-924. doi:https://doi.org/10. 1136/pgmj.62.732.919

23. Chrysant S, "The Lisinopril-Hydrochlorothiazide Group." Antihypertensive effectiveness of low-dose lisinopril-hydrochlorothiazide combination: a large multicenter study. Arch Intern Med. 1994;154:737-743.

24. Maroko P, McDevitt J, Fox M, et al. Antihypertensive effectiveness of very low doses of hydrochlorothiazide: results of the PHICOG trial. Clin Ther. 1989;11(1):94-119.

25. Pool PE, Applegate WB, Woehler T, Sandall P, Cady WJ. A Randomized, Controlled Trial Comparing Diltiazem, Hydrochlorothiazide, and Their Combination in the Therapy of Essential Hypertension. Pharmacotherapy. 1993;13(5):487-493. doi:https://doi.org/10.1002/j.18759114.1993.tb04312.x

26. Reid IR, Ames RW, Orr-Walker BJ, et al. Hydrochlorothiazide Reduces Loss of Cortical Bone in Normal Postmenopausal Women: A Randomized Controlled Trial. Am J Med. 2000; 109:362-370.

27. Savage PJ, Pressel SL, Curb JD, et al. Influence of Long-term, LowDose, Diuretic-Based, Antihypertensive Therapy on Glucose, Lipid, Uric Acid, and Potassium Levels in Older Men and Women With Isolated Systolic Hypertension The Systolic Hypertension in the Elderly Program. Arch Intern Med. 1998;158:741-751. http://archinte.jamanetwork. com/.

28. Siegel D, Saliba P, Haffner S. Glucose and insulin levels during diuretic therapy in hypertensive men. Hypertension. 1994;23:688-694. doi:https://doi.org/10.1161/01.hyp.23.6.688

29. Damian DJ, McNamee R, Carr M. Changes in selected metabolic parameters in patients over 65 receiving hydrochlorothiazide plus amiloride, atenolol or placebo in the MRC elderly trial. BMC Cardiovasc Disord. 2016;16:188. doi:https://doi.org/10.1186/s12872-016-0368-2

30. Fiddes R, Blumenthal J, Dawson JE, et al. Evaluation of indapamide $1.25 \mathrm{mg}$ once daily in elderly patients with mild to moderate hypertension. J Hum Hypertens. 1997;11:239-244. doi:https://doi.org/10. 1038/sj.jhh. 1000419

31. Goldman AI, Steele BW, Schaper HW, Fitz AE, Frohlich ED, Perry HMJ. Serum lipoprotein levels during chlorthalidone therapy. A Veterans Administration-National Heart, Lung, and Blood Institute 
Cooperative Study on Antihypertensive Therapy: Mild Hypertension. JAMA. 1980;224(15):1691-1695.

32. Grimm RH, Leon AS, Hunninghake DB, Lenz $K$, Hannan P, Blackburn $\mathbf{H}$. Effects of thiazide diuretics on plasma lipids and lipoproteins in mildly hypertensive patients. A double-blind controlled trial. Ann Intern Med. 1981;94:7-11. doi:https://doi.org/10.1109/NBiS.2011.113

33. Jounela AJ, Lilja M, Lumme J, et al. Relation Between Low Dose of Hydrochlorothiazide, Antihypertensive Effect and Adverse Effects. Blood Press. 1994;3:231-235.

34. Koh KK, Quon MJ, Han SH, et al. Distinct vascular and metabolic effects of different classes of antihypertensive drugs. Int $J$ Cardiol. 2010;140:73-81.

35. Lambers Heerspink HJ, De Zeeuw D, Wie L, Leslie B, List $\mathbf{J}$ Dapagliflozin a glucose-regulating drug with diuretic properties in subjects with type 2 diabetes. Diabetes, Obes Metab. 2013;15:853862. doi:https://doi.org/10.1111/dom.12127

36. Malekzadeh F, Marshall T, Pourshas A, et al. A pilot double-blind randomised placebo controlled trial of the effects of fixed-dose combination therapy ('polypill') on cardiovascular risk factors. Int $J$ Clin Pract. 2010;64(9):1120-1227. doi:https://doi.org/10.1111/j.1742-1241. 2010.02412.x

37. Berglund G, Andersson O. Beta-blockers or diuretics in hypertension? A six-year follow-up of blood pressure and metabolic side effects. Lancet. 1981:744-747.

38. Bichisao E, Pollavini G, Alberti D, Baroni L, Cortellaro M. Slowrelease metoprolol and chlorthalidone in the management of arterial hypertension: a multicentre study. Curr Ther Res. 1984;36(3):522-529.

39. Skoczylas A, Piecha G, Wi \cek A. Effects of antihypertensive treatment on plasma apelin, resistin, and visfatin concentrations. Pol Arch Med Wewn. 2016;126(4):243-253. doi:https://doi.org/10.20452/pamw. 3390

40. Smith SM, Gong Y, Turner ST, et al. Blood pressure responses and metabolic effects of hydrochlorothiazide and atenolol. Am J Hypertens. 2012;25(3):359-365. doi:https://doi.org/10.1038/ajh.2011.215

41. Yonga G, Ogola E, Orinda D. Metabolic effects of propranolol and hydroflumethiazide treatment in Kenyans with mild to moderate hypertension. East Afr Med J. 1993;70(11):696.

42. Fogari R, Zoppi A, Malamani G, Marasi G, Vanasia A, Villa G. Effects of different antihypertensive drugs on plasma fibrinogen in hypertensive patients. Br J Clin Pharmacol. 1995;39:471-476. doi:https://doi.org/ 10.1111/j.1365-2125.1995.tb04482.x

43. Veterans Administration Cooperative Study Group on Antihypertensive Agents. Efficacy of nadolol alone and combined with bendroflumethiazide and hydralazine for systemic hypertension. Am $J$ Cardiol. 1983;52:1230-1237

44. Milon H, Froment A, Gaspard P, Delahaye J. Treatment of arterial hypertension: A comparative trial of atenolol versus chlorthalidone. Clin Trials J. 1984;6:298-307.

45. Monmany J, Djomingo P, Gomez JA, et al. Effects of long-term treatment with metoprolol and hydrochlorothiazide on plasma lipids and lipoproteins. J Intern Med. 1990;228:323-331. doi:https://doi.org/ 10.1111/j.1365-2796.1990.tb00240.x

46. Os I, Hotnes T, Dollerup J, Mogensen CE. Comparison of the combination of enalapril and a very low dose of hydrochlorothiazide with atenolol in patients with mild-to moderate hypertension. Am J Hypertens. 1997;10:889-904. doi:https://doi.org/10.1016/S08957061(97)00110-6

47. Pollavini G, Comi D, Grillo C, et al. Multicentre randomized cross-over double-blind comparison between chlorthalidone and slow-release oxprenolol in mild-to-moderate hypertension. Curr Ther Res. 1984;35(3):467-475.

48. Rajzer M, Wojciechowska W, Kameczura T, et al. The effect of antihypertensive treatment on arterial stiffness \& serum concentration of selected matrix metalloproteinases. Arch Med Sci. 2017;13(4):760770. doi:https://doi.org/10.5114/aoms.2016.58825

49. Veterans Administration Cooperative Study Group on Antihypertensive Agents. Propranolol or hydrochlorothiazide alone for the initial treatment of hypertension: IV. Effect on Plasma Glucose and Glucose Tolerance. Hypertension. 1985;7(6):1008-1016.

50. Rasmussen S, Borrild N, Vang Andersen J. Efficacy and safety of 24 weeks of therapy with bendroflumethiazide $1.25 \mathrm{mg} /$ day or $2.5 \mathrm{mg} /$ day and potassium chloride compared with enalapril $10 \mathrm{mg} /$ day and amlodipine $5 \mathrm{mg} /$ day in patients with mild to moderate primary hypertension a multicentre, randomized, open study. Clin Drug Investig. 2006;26(2):91-101. doi:https://doi.org/10.2165/00044011200626020-00004
51. Takihata M, Nakamura A, Kondo Y, Kawasaki S, Kimura M, Terauchi Y. Comparison of azelnidipine and trichlormethiazide in Japanese type 2 diabetic patients with hypertension:The COAT randomized controlled trial. PLoS One. 2015. doi:https://doi.org/10.1371/journal.pone. 0125519

52. Bosone D, Costa A, Ghiotto N, et al. Effect of ramipril/ hydrochlorothiazide and ramipril/canrenone combination on atrial fibrillation recurrence in hypertensive type 2 diabetic patients with and without cardiac autonomic neuropathy. Arch Med Sci. 2017;13(3):550-557. doi:https://doi.org/10.5114/aoms.2016.62448

53. Byington RP, Furberg CD, Craven TE, Pahor M, Sowers JR. Isradipine in Prediabetic Hypertensive Subjects. Diabetes Care. 1998;21:2103-2110.

54. Calvo C, Gude F, Abellán J, et al. A comparative evaluation of amlodipine and hydrochlorothiazide as monotherapy in the treatment of isolated systolic hypertension in the elderly. Clin Drug Investig. 2000;19(5):317-326. doi:https://doi.org/10.2165/00044011200019050-00001

55. Leehey D, Hartman E. Comparison of diltiazem and hydrochlorothiazide for treatment of patients 60 years of age or older with systemic hypertension. Am J Cardiol. 1988;62(17):1218-1223.

56. Garg R, Rao AD, Baimas-George M, et al. Mineralocorticoid receptor blockade improves coronary microvascular function in individuals with type 2 diabetes. Diabetes. 2015;64:236-242. doi:https://doi.org/10. 2337/db14-0670

57. Haenni A, Andersson $\mathbf{P}$, Lind L, Berne C, Lithell $\mathbf{H}$. Electrolyte changes and metabolic effects of lisinopril/bendrofluazide treatment. Results from a randomized, double-blind study with parallel groups. Am $J$ Hypertens. 1994;7:615-622.

58. Lin M, Yang Y-F, Chiang Derek Lee H-T, Wang S-P, Chang M-S, Cheitlin MD. Beneficial Effects of Angiotensin-Converting Enzyme Inhibitors on Cardiovascular and Renal Functions in Patients with Hypertension and Diabetes. Acta Cardiol Sin. 1995;11:30-38.

59. Lindholm LH, Persson M, Alaupovic P, Carlberg B, Svensson A, Samuelsson O. Metabolic outcome during 1 year in newly detected hypertensives: results of the Antihypertensive Treatment and Lipid Profile in a North of Sweden Efficacy Evaluation (ALPINE study). $J$ Hypertens. 2003;21:1563-1574. doi:https://doi.org/10.1097/01.hjh. 0000084723.53355 .76

60. Makita S, Abiko A, Naganuma Y, Tamada M, Nakamura M. Efficacy of low-dose hydrochlorothiazide in combination with telmisartan on early morning blood pressure in uncontrolled hypertensive patients. Clin Exp Hypertens. 2009;31:105-115. doi:https://doi.org/10.1080/ 10641960802621259

61. Marre M, Puig JG, Kokot F, et al. Equivalence of indapamide SR and enalapril on microalbuminuria reduction in hypertenisve patients with type 2 diabetes: the NESTOR study. J Hypertens. 2004;22:1613-1622. doi:https://doi.org/10.1097/01.hjh.0000133733.32125.09

62. Nishimura $\mathbf{H}$, Shintani $\mathbf{M}$, Maeda $\mathbf{K}$, et al. Which is a better treatment for hypertensive patients with diabetes: A combination of losartan and hydrochlorothiazide or a maximum dose of Losartan? Clin Exp Hypertens. 2013;35(8):582-588. doi:https://doi.org/10.3109/10641963. 2013.776564

63. Pollare T, Lithell H, Berne C. A comparison of the effects of hydrochlorothiazide and captopril on glucose and lipid metabolism in patients with hypertension. N Engl J Med. 1989;321(13):868-873.

64. Roman M, Alderman M, Pickering T, et al. Differential effects of angiotensin converting enzyme inhibition and diuretic therapy on reductions in ambulatory blood pressure. Am $J$ Hypertens. 1998; 11(4):387-396.

65. Rosenthal T, Grossman E, Rathaus M, et al. Treatment of hypertension by enalapril and hydrochlorothiazide separately and together: a multicenter study. Isr J Med Sci. 1990;26(2):63-66.

66. Scali M, Armanini D, Mantero F, et al. Metabolic effects of lisinopril versus hydrochlorothiazide plus amiloride in essential hypertension. Curr Ther Res. 1992;52(3):397-405. doi:https://doi.org/10.1016/ S0011-393X(05)80414-X

67. Stimpel M, Koch B, Oparil S. Antihypertensive treatment in postmenopausal women: results from a prospective, randomized, double-blind, controlled study comparing an ACE inhibitor (moexipril) with a diuretic (hydrochlorothiazide). Cardiology. 1998;89(4):271-276.

68. Zappe DH, Sowers JR, Hsueh WA, et al. Metabolic and antihypertensive effects of combined angiotensin receptor blocker and diuretic therapy in prediabetic hypertensive patients with the cardiometabolic syndrome. J Clin Hypertens. 2008;10:894-903. doi:https://doi.org/10. $1111 /$ j.1751-7176.2008.00054.x 
69. Zhang J-L, Gin Y-W, Zheng X, Giu J-L, Zhao X-X, Zou D-J. Combination therapy with angiotensin-converting enzyme inhibitors and indapamide impairs glucose tolerance in Chinese hypertensive patients. Blood Press. 2010;19:110-118.

70. Zhang $\mathbf{P}$, Wang $\mathbf{H}$, Sun $\mathbf{L}$, et al. Telmisartan and hydrochlorothiazide antihypertensive treatment in high sodium intake population: A randomized double-blind trial. J Hypertens. 2017;35(10):2077-2085. doi:https://doi.org/10.1097/HJH.0000000000001407

71. Abe M, Okada K, Maruyama T, Matsumoto K. Antiproteinuric and blood pressure-lowering effects of a fixed-dose combination of losartan and hydrochlorothiazide in hypertensive patients with stage 3 chronic kidney disease. Pharmacotherapy. 2009;29(9):1061-1072.

72. Brandao SA, Izar MC, Fischer SM, et al. Early increase in autoantibodies against human oxidized low-density lipoprotein in hypertensive patients after blood pressure control. Am J Hypertens. 2010;23(2):208214

73. Fonseca HAR, Fonseca FA, Lins LC, et al. Antihypertensive therapy increases natural immunity response in hypertensive patients. Life Sci. 2015;143:124-130. doi:https://doi.org/10.1016/j.lfs.2015.10.030

74. Fuchs FD, Scala LCN, Vilela-Martin JF, et al. Effectiveness of chlorthalidone/amiloride versus losartan in patients with stage hypertension: Results from the PREVER-treatment randomized trial. $J$ Hypertens. 2016;34:798-806. doi:https://doi.org/10.1097/HJH. 0000000000000837

75. Brown MJ, Williams B, Morant S V., et al. Effect of amiloride, or amiloride plus hydrochlorothiazide, versus hydrochlorothiazide on glucose tolerance and blood pressure (PATHWAY-3): A parallel-group, double-blind randomised phase 4 trial. Lancet Diabetes Endocrinol. 2016;4:136-147. doi:https://doi.org/10.1016/S2213-8587(15)00377-

76. Multicenter Diuretic Cooperative Study Group." Multiclinic Comparison of Amiloride, Hydrochlorothiazide, and Hydrochlorothiazide Plus Amiloride in Essential Hypertension. Arch Intern Med. 1981;141:482486.

77. Momeni A, Behradmanesh MS, Kheiri S, Karami Horestani M Evaluation of spironolactone plus hydrochlorothiazide in reducing proteinuria in type 2 diabetic nephropathy. $J$ Renin-AngiotensinAldosterone Syst. 2015;16(1):113-118. doi:https://doi.org/10.1177/ 1470320313481485

78. Yutaka M, Mifune M, Kubota E, Itoh H, Saito I. Comparison of effects of low dose of spironolactone and a thiazide diuretic in patients with hypertension treated with an angiotensin-converting enzyme inhibitor or an angiotensin type 1 receptor blocker. Clin Exp Hypertens. 2009;31:648-656. doi:https://doi.org/10.3109/10641960903407017

79. Amin NB, Wang X, Mitchell JR, Lee DS, Nucci G, Rusnak JM. Blood pressure-lowering effect of the sodium glucose co-transporter-2 inhibitor ertugliflozin, assessed via ambulatory blood pressure monitoring in patients with type 2 diabetes and hypertension. Diabetes, Obes Metab. 2015;17:805-808. doi:https://doi.org/10.1111/dom. 12486

80. Campo C, Segura J, Roldán C, Alcázar JM, Rodicio JL, Ruilope LM Doxazosin GITS versus hydrochlorothiazide as addon therapy in patients with uncontrolled hypertension. Blood Press. 2003;12(Suppl. 2):16-21. doi:https://doi.org/10.1080/08038020310016369

81. Charansonney $\mathbf{O}$, Lievre $\mathbf{M}$, Laville $\mathbf{M}$, et al. The EUREVIE Study: Contrasting effect of piretanide and thiazides in mild to moderate hypertension. Therapie. 1997;52(3):169-177.

82. Veterans Administration Cooperative Study Group on Antihypertensive Agents. Comparative effects of ticrynafen and hydrochlorothiazide in the treatment of hypertension. N Engl J Med. 1979;301(6):293-297.

83. Hegbrant J, Skogstrom K, Mansby J. Comparison of slow-release piretanide and bendroflumethiazide in the treatment of mild to moderate hypertension. J Int Med Res. 1989;17(5):426-434.

84. Helgeland A, Leren P, Foss O, Hjermann I, Holme I, Lund-Larsen P. Serum glucose levels during long-term observation of treated and untreated men with mild hypertension. The OSLO study. Am J Med. 1984;76:802-805.

85. Obel A, Griffin L, Were J. Comparison of slow-release frusemide and bendrofluazide in the treatment of moderate hypertension in Kenyan negroes. Clin Trials J. 1984;6:443-450.

86. Ueda $\mathbf{S}$, Morimoto $\mathbf{T}$, Ando $\mathbf{S}$, et al. A randomised controlled trial for the evaluation of risk for type 2 diabetes in hypertensive patients receiving thiazide diuretics: Diuretics In the Management of Essential hypertension (DIME) study. BMJ Open. 2014;4(7):4576-4576. doi:https://doi.org/10.1136/bmjopen-2013-004576
87. Wicker P, Clementy J. Comparison of the effects of muzolimine and a fixed combination of diuretics in essential hypertension. Clin Pharmacol Ther. 1986;39:537-542. doi:https://doi.org/10.1038/clpt. 1986.92

88. Fogari R, Derosa G, Zoppi A, et al. Effect of Delapril/Manidipine vs Olmesartan/ Hydrochlorothiazide Combination on Insulin Sensitivity and Fibrinogen in Obese Hypertensive Patients. Intern Med. 2008;47:361-366. doi:https://doi.org/10.2169/internalmedicine.47. 0449

89. Fogari R, Derosa G, Zoppi A, Lazzari P, D'Angelo A, Mugellini A. Comparative effect of canrenone or hydrochlorothiazide addition to valsartan/amlodipine combination on urinary albumin excretion in well-controlled type 2 diabetic hypertensive patients with microalbuminuria. Expert Opin Pharmacother. 2014;15(4):453-459. doi:https://doi. org/10.1517/14656566.2014.874415

90. Ghiadoni L, Bruno RM, Cartoni G, et al. Combination therapy with lercanidipine and enalapril reduced central blood pressure augmentation in hypertensive patients with metabolic syndrome. Vascul Pharma col. 2017;92:16-21. doi:https://doi.org/10.1016/j.vph.2015.06.004

91. Holzgreve H, Nakov R, Beck K, Janka HU. Antihypertensive therapy with verapamil SR plus trandolapril versus atenolol plus chlorthalidone on glycemic control. Am J Hypertens. 2003;16:381-386. doi:https:// doi.org/10.1016/S0895-7061(03)00062-1

92. Karashima S, Yoneda T, Kometani M, et al. Angiotensin II receptor blocker combined with eplerenone or hydrochlorothiazide for hypertensive patients with diabetes mellitus. Clin Exp Hypertens. 2016;37(7):565-570. doi:https://doi.org/10.3109/10641963.2016. 1151526

93. Kato J, Yokota $\mathbf{N}$, Tamaki $\mathbf{N}$, et al. Comparison of combination therapies, including the angiotensin receptor blocker olmesartan and either a calcium channel blocker or a thiazide diuretic, in elderly patients with hypertension. Hypertens Res. 2011;34:331-335. doi:https://doi.org/10.1038/hr.2010.233

94. Lee I-T, Hung Y-J, Chen J-F, Wang C-Y, Lee W-J, Huey-Herng Sheu W. Comparison of the efficacy and safety profiles of two fixed- dose combinations of antihypertensive agents, amlodipine/benazepril versus valsartan/hydrochlorothiazide, in patients with type 2 diabetes mellitus and hypertension: A 16-week, multicenter, tand. Clin Ther 2012;34(8):1735-1750. doi:https://doi.org/10.1016/j.clinthera.2012. 06.014

95. Martinez-Martin FJ, Rodriguez-Rosas H, Peiro-Martinez I, SorianoPerera P, Pedrianes-Martin P, Comi-Diaz C. Olmesartan/amlodipine vs olmesartan/hydrochlorothiazide in hypertensive patients with metabolic syndrome: The OLAS study. J Hum Hypertens. 2011;25:346-353. doi:https://doi.org/10.1038/jhh.2010.104

96. Matsui Y, Eguchi K, Ishikawa J, Shimada K, Kario K. Urinary albumin excretion during angiotensin II receptor blockade: Comparison of combination treatment with a diuretic or a calcium-channel blocker. Am J Hypertens. 2011;24(4):466-473. doi:https://doi.org/10.1038/ajh. 2010.240

97. Mugellini A, Preti P, Zoppi A, et al. Effect of delapril-manidipine combination vs irbesartan-hydrochlorothiazide combination on fibrinolytic function in hypertensive patients with type II diabetes mellitus. $J$ Hum Hypertens. 2004; 18:687-691. doi:https://doi.org/10.1038/sj.jhh. 1001726

98. Nielsen S, Schmitz A, Mogensen CE, Knudsen RE, Dollerup J. Enalapril versus bendroflumethiazide in type 2 diabetes complicated by hypertension. QJM. 1994;87:747-754. doi:https://doi.org/10.1093/ oxfordjournals.qjmed.a068893

99. Nishiwaki M, Hosoai H, Ikewaki $\mathbf{K}$, et al. Efficacy and Effects on Lipid Metabolism of Combination Treatment With Losartan + Hydrochlorothiazide Versus Losartan + Amlodipine: A 48-Week Prospective, Multicenter, Randomized, Open-Label Trial, Clin Ther. 2013;35(4):461-473. doi:https://doi.org/10.1016/j.clinthera.2013.02.021

100. Oshikawa J, Toya Y, Morita S, et al. Angiotensin receptor blocker (ARB)-diuretic versus ARB-calcium channel blocker combination therapy for hypertension uncontrolled by ARB monotherapy. Clin Exp Hypertens. 2014;36(4):244-250. doi:https://doi.org/10.3109/ 10641963.2013 .810227

101. Pareek A, Karnik N, Salagre SB, et al. Clinical effectiveness of low-dose chlorthalidone $(6.25 \mathrm{mg})+$ atenolol combination in stage I hypertensive patients: a multicenter, randomized, controlled study. Curr Med Res Opin. 2008;24(6):1771-1779. doi:https://doi.org/10.1185/ 03007990802118071

102. Posadzy-Malaczynska A, Rajpold K, Woznicka-Leskiewicz L, Marcinkowska J. Hemodynamic and metabolic effects of estrogen plus progestin therapy in hypertensive postmenopausal women treated with 
an ACE-inhibitor or a diuretic. Clin Res Cardiol. 2014;104:38-50. doi:https://doi.org/10.1007/s00392-014-0755-6

103. Saruta T, Ogihara T, Saito I, et al. Comparison of olmesartan combined with a calcium channel blocker or a diuretic in elderly hypertensive patients (COLM Study): Safety and tolerability. Hypertens Res. 2015;38:132-136. doi:https://doi.org/10.1038/hr.2014.141

104. Tani S, Asayama K, Oiwa $\mathbf{K}$, et al. The effects of increasing calcium channel blocker dose vs. adding a diuretic to treatment regimens for patients with uncontrolled hypertension. Hypertens Res. 2017;40:892898. doi:https://doi.org/10.1038/hr.2017.56

105. Bakris G, Molitch M, Hewkin A, et al. Differences in glucose tolerance between fixed-dose antihypertensive drug combinations in people with metabolic syndrome. Diabetes Care. 2006;29:2592-2597. doi:https:// doi.org/10.2337/dc06-1373

106. Christogiannis LG, Kostapanos MS, Tellis CC, Milionis HJ, Tselepis AD, Elisaf MS. Distinct effects of fixed combinations of valsartan with either amlodipine or hydrochlorothiazide on lipoprotein subfraction profile in patients with hypertension. J Hum Hypertens. 2013;27:4450. doi:https://doi.org/10.1038/jhh.2011.108

107. Deedwania P, Shea J, Chen W, Brener L. Effects of Add-On Nebivolol on Blood Pressure and Glucose Parameters in Hypertensive Patients With Prediabetes. J Clin Hypertens. 2013;15:270-278. doi:https://doi. org/10.1111/jch.12071

108. Derosa G, Querci F, Franzetti I, Dario Ragonesi P, D'Angelo A, Maffioli P. Comparison of the effects of barnidipine+losartan compared with telmisartan+hydrochlorothiazide on several parameters of insulin sensitivity in patients with hypertension and type 2 diabetes mellitus. Hypertens Res. 2015;38:690-694. doi:https://doi.org/10.1038/hr. 2015.57

109. Ferdinand KC, Weitzman R, Israel M, Lee J, Purkayastha D, Jaimes EA. Efficacy and safety of aliskiren-based dual and triple combination therapies in US minority patients with stage 2 hypertension. J Am Soc Hypertens. 2011;5(2):102-113. doi:https://doi.org/10.1016/j.jash. 2011.01.006

110. Fogari R, Corradi L, Zoppi A, et al. Addition of manidipine improves the antihypertensive effect of candesartan hypertensive patients with type II diabetes. Am J Hypertens. 2007;20:1092-1096.

111. Fogari R, Derosa G, Zoppi A, et al. Effects of Manidipine/Delapril versus Olmesartan/Hydrochlorothiazide Combination Therapy in Elderly Hypertensive Patients with Type 2 Diabetes Mellitus. Hypertens Res. 2008;31:43-50. doi:https://doi.org/10.1291/hypres.31.43

112. MRC Working Party. Medical Research Council trial of treatment of hypertension in older adults: principal results MRC Working Party. BMJ. 1992;304:405-412.

113. Helgeland A. Treatment of mild hypertension: A five year controlled drug trial: The Oslo study. Am J Med. 1980;69(5):725-732. doi:https:// doi.org/10.1016/0002-9343(80)90438-6

114. Durán-Salgado MB, Garro-Almendaro AKA, Rubio-Guerra AF. Cambios metabólicos ocasionados por las combinaciones de losartán con hidroclorotiazida o con amlodipino en pacientes hipertensos. Med Interna Mex. 2015;31:395-401.
115. Ogihara T, Saruta T, Rakugi H, et al. Combinations of olmesartan and a calciumchannel blocker or a diuretic inelderly hypertensive patients: A randomized, controlled trial. J Hypertens. 2014;32(10):2054-2063. doi:https://doi.org/10.1097/HJH.0000000000000281

116. "SHEP Cooperative Research Group." Prevention of Stroke by Antihypertensive Drug Treatment in Older Persons With Isolated Systolic Hypertension. JAMA. 1991;265:3255-3264.

117. Siegel D, Hulley SB, Black DM, et al. Diuretics, serum and intracellular electrolyte levels, and ventricular arrhythmias in hypertensive men. JAMA. 1992;267(8): 1083-1089.

118. Veterans Administration Cooperative Study Group on Antihypertensive Agents. Comparison of propranolol and hydrochlorothiazide for the initial treatment of hypertension. II. Results of long-term therapy. Veterans Administration Cooperative Study Group on Antihypertensive Agents. JAMA. 1982;248(16):1996-2003. doi:https://doi.org/10.1001/ jama.248.16.2004

119. Glasziou P, Sanders S. Investigating causes of heterogeneity in systematic reviews. Stat Med. 2002;21(11):1503-1511. doi:https://doi. org/10.1002/sim.1183

120. Peterzan MA, Hardy R, Chaturvedi N, Hughes AD. Meta-analysis of dose-response relationships for hydrochlorothiazide, chlorthalidone, and bendroflumethiazide on blood pressure, serum potassium, and urate. Hypertension. 2012;59(6):1104-1109. doi:https://doi.org/10. 1161/HYPERTENSIONAHA.111.190637

121. Shafi T, Appel LJ, Miller ER, Klag MJ, Parekh RS. Changes in serum potassium mediate thiazide-induced diabetes. Hypertension. 2008;52(6):1022-1029. doi:https://doi.org/10.1161/HYPERTENSIONAHA. 108.119438

122. Moore MJ, Gong Y, Hou W, et al. Predictors for glucose change in hypertensive participants following short-term treatment with atenolol or hydrochlorothiazide. Pharmacotherapy. 2014;34(11):1132-1140. doi:https://doi.org/10.1002/phar. 1483

123. Verdecchia P, Reboldi G, Angeli F, et al. Adverse Prognostic Significance of New Diabetes in Treated Hypertensive Subjects. Hypertension. 2004;43(5):963-969. doi:https://doi.org/10.1161/01.HYP. 0000125726.92964.ab

124. Lin J., Chang H., Ku C., Chen H., Ku C. Hydrochlorothiazide hypertension treatment induced metabolic effects in type 2 diabetes: a meta-analysis of parallel-design RCTs. Eur Rev Med Pharmacol Sci. 2016;20:2926-2934.

125. Zhang $\mathbf{X}$, Zhao $\mathbf{Q}$. Association of thiazide-type diuretics with glycemic changes in hypertensive patients: A systematic review and metaanalysis of randomized controlled clinical trials. $J$ Clin Hypertens (Greenwich). 2015;18(4):1-10. doi:https://doi.org/10.1111/jch.12679

126. Sterne JAC, Sutton AJ, Ioannidis JPA, et al. Recommendations for examining and interpreting funnel plot asymmetry in meta-analyses of randomised controlled trials. BMJ. 2011;343(7818): 1-8. doi:https://doi. org/10.1136/bmj.d4002

Publisher's Note Springer Nature remains neutral with regard to jurisdictional claims in published maps and institutional affiliations. 